\title{
Investigation on the interaction mechanism of the solvent extraction for mercaptan removal from LPG
}

\author{
Dexin Sun${ }^{1}$, Yulong Zhao ${ }^{1}$, Yanjing Cao ${ }^{1}$, Mengqi Liu ${ }^{1}$, Yuxiang \\ Zhang $^{1}$, Liang Zhao ${ }^{1 *}$, Yuhao Zhang ${ }^{1}$, JinsenGao ${ }^{1}$, ChunmingXu${ }^{1}$, \\ Tianzhen $\mathrm{Hao}^{2}$, Jialing Chen ${ }^{3}$, and Dewei $\mathrm{Ji}^{4}$
}

${ }^{1}$ State Key Laboratory of Heavy Oil Processing, China University of Petroleum (Beijing), Beijing 102249, People's Republic of China

${ }^{2}$ Hebei Jingzhi Technology Co., LTD, He bei 061000, People's Republic of China ${ }^{3}$ PetroChina Qinghai Oilfield Company, Qinghai 817500, People's Republic of China ${ }^{4}$ PetroChina Dagang Petrochemical Company, Tianjin 300280, People's Republic of China

Email: Liang Zhao(liangzhao@cup.edu.cn)

Tel: $\quad 86-10-89739078$

Fax: $\quad 86-10-697247$ 
Table S1. The Information of Simulated Systems

Table S2. Height and location of first peak for the RDFs between PM molecular with all types of solvent

Fig.1S. The most stable structures with minimum energy for (a) NPA-PM; (b) IPA-PM; (c) ET-PM; (d) MEA-PM;(e) MeOH-PM; (f) EG-PM dimers.

Fig.2S. Plot of $\operatorname{sign}\left(\lambda_{2}\right) \rho$ vs RDG for (a) NPA-PM; (b) IPA-PM; (c) ET-PM; (d) MEA-PM;(e) MeOH-PM; (f) EG-PM dimers.

Fig.3S. Gradient isosurfaces for (a) NPA-PM; (b) IPA-PM; (c) ET-PM; (d) MEA-PM;(e) MeOH-PM; (f) EG-PM dimers.

Fig. 4S. AIM molecular topography map showing BCPs distribution and bond paths for (a) NPA PM; (b) IPA - PM; (c) ET - PM; (d) MEA.-PM;(e) MeOH-PM; (f) EG-PM. Orange dots represent BCPs. 
Table S1. The Information of Simulated Systems

\begin{tabular}{|c|c|c|c|c|c|c|c|c|c|}
\hline System & $\begin{array}{l}\text { Organic } \\
\text { solvent }\end{array}$ & Count & Water & Count & Mercaptan & Count & $\begin{array}{c}\text { Calculated } \\
\text { density } \\
\left(\mathrm{g} / \mathrm{cm}^{3}\right) \\
\end{array}$ & $\begin{array}{c}\text { Experimental } \\
\text { density } \\
\left(\mathrm{g} / \mathrm{cm}^{3}\right)\end{array}$ & $\begin{array}{c}\text { Density } \\
\text { error }\end{array}$ \\
\hline PM-NPA- $\mathrm{H}_{2} \mathrm{O}$ & NPA & 52 & $\mathrm{H}_{2} \mathrm{O}$ & 1500 & PM & 10 & 0.972 & 0.987 & $-1.51 \%$ \\
\hline PM-ACTN- $\mathrm{H}_{2} \mathrm{O}$ & ACTN & 53 & $\mathrm{H}_{2} \mathrm{O}$ & 1500 & PM & 10 & 0.982 & 0.982 & $0.02 \%$ \\
\hline PM-IPA- $\mathrm{H}_{2} \mathrm{O}$ & IPA & 52 & $\mathrm{H}_{2} \mathrm{O}$ & 1500 & PM & 10 & 0.976 & 0.981 & $-0.50 \%$ \\
\hline PM-ET- $\mathrm{H}_{2} \mathrm{O}$ & ET & 67 & $\mathrm{H}_{2} \mathrm{O}$ & 1500 & $\mathrm{PM}$ & 10 & 0.975 & 0.980 & $-0.51 \%$ \\
\hline PM-MEA- $\mathrm{H}_{2} \mathrm{O}$ & MEA & 51 & $\mathrm{H}_{2} \mathrm{O}$ & 1500 & PM & 10 & 1.007 & 0.998 & $0.94 \%$ \\
\hline PM-EG- $\mathrm{H}_{2} \mathrm{O}$ & $\mathrm{EG}$ & 50 & $\mathrm{H}_{2} \mathrm{O}$ & 1500 & PM & 10 & 1.052 & 1.008 & $4.37 \%$ \\
\hline $\mathrm{PM}-\mathrm{MeOH}-\mathrm{H}_{2} \mathrm{O}$ & $\mathrm{MeOH}$ & 96 & $\mathrm{H}_{2} \mathrm{O}$ & 1500 & PM & 10 & 0.971 & 0.981 & $-1.03 \%$ \\
\hline PM-IBAK- $\mathrm{H}_{2} \mathrm{O}$ & IBAK & 37 & $\mathrm{H}_{2} \mathrm{O}$ & 1500 & PM & 10 & 1.031 & 1.035 & $-0.40 \%$ \\
\hline PM-HACK - $\mathrm{H}_{2} \mathrm{O}$ & HACK & 56 & $\mathrm{H}_{2} \mathrm{O}$ & 1500 & PM & 10 & 1.064 & 1.088 & $-2.16 \%$ \\
\hline $\mathrm{PM}-\mathrm{H}_{2} \mathrm{O}$ & - & - & $\mathrm{H}_{2} \mathrm{O}$ & 1600 & PM & 10 & 0.996 & 1.001 & $-0.50 \%$ \\
\hline
\end{tabular}


Table S2. Height and location of first peak for the RDFs between PM molecular with all types of

\begin{tabular}{cccc}
\multicolumn{4}{c}{ solvent } \\
\hline System & RDF & $\mathrm{r}$ & $\mathrm{g}(\mathrm{r})$ \\
\hline PM-NPA- $\mathrm{H}_{2} \mathrm{O}$ & PM-NPA & 5.31 & 6.64 \\
PM-ACTN-H $\mathrm{H}_{2} \mathrm{O}$ & PM-ACTN & 5.13 & 5.89 \\
PM-IPA- $\mathrm{H}_{2} \mathrm{O}$ & PM-IPA & 5.31 & 3.75 \\
PM-ET- $\mathrm{H}_{2} \mathrm{O}$ & PM-ET & 4.97 & 3.29 \\
PM-MEA- ${ }_{2} \mathrm{O}$ & PM-MEA & 5.09 & 2.59 \\
PM-EG- $\mathrm{H}_{2} \mathrm{O}$ & PM-EG & 5.13 & 2.23 \\
PM-MeOH- $\mathrm{H}_{2} \mathrm{O}$ & PM-MeOH & 4.55 & 2.15 \\
PM-IBAK- $\mathrm{H}_{2} \mathrm{O}$ & PM-IBAK & 6.05 & 1.99 \\
PM-HACK - $\mathrm{H}_{2} \mathrm{O}$ & PM-HACK & 5.31 & 1.31 \\
PM- $\mathrm{H}_{2} \mathrm{O}$ & PM- $\mathrm{H}_{2} \mathrm{O}$ & 4.37 & 1.65 \\
\hline
\end{tabular}




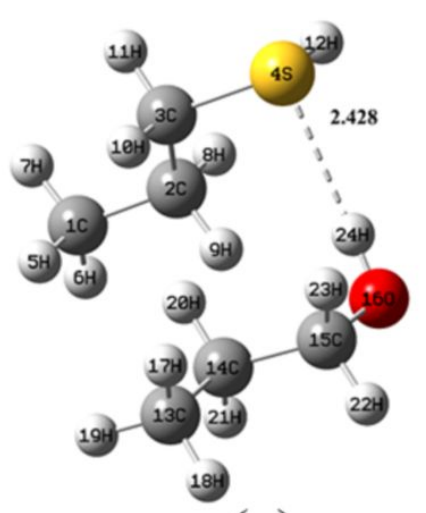

(a)

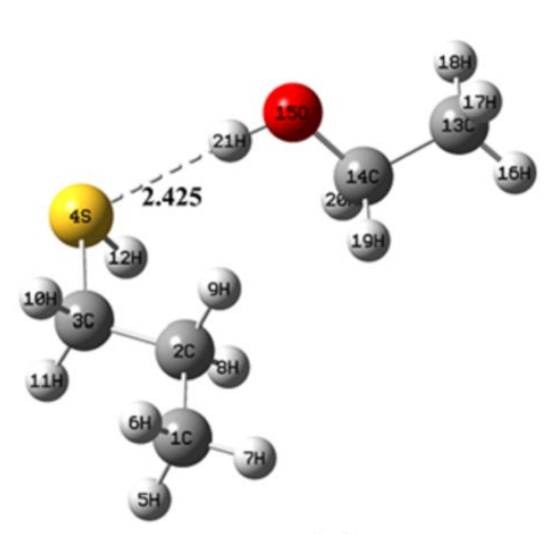

(c)

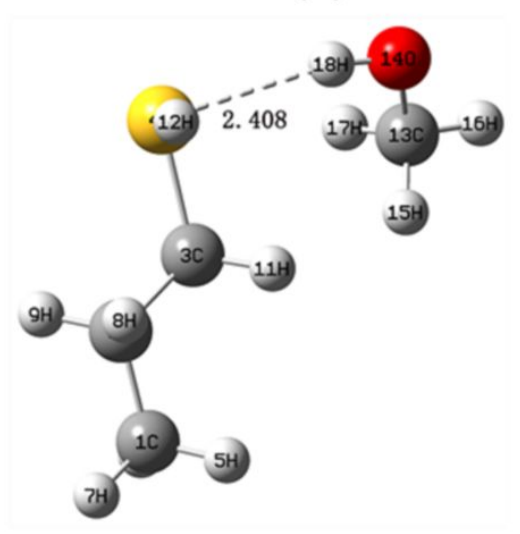

(e)

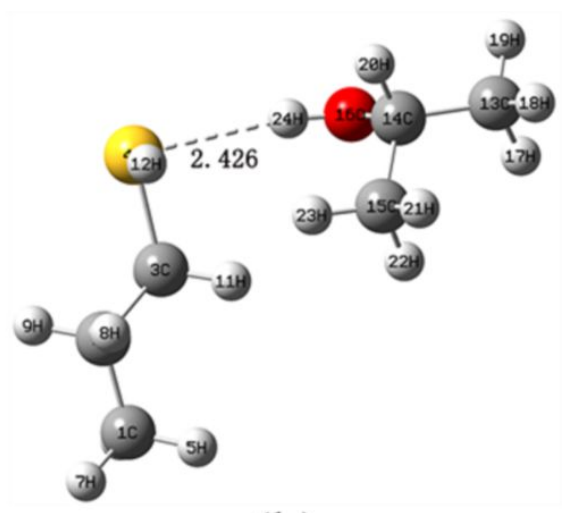

(b)

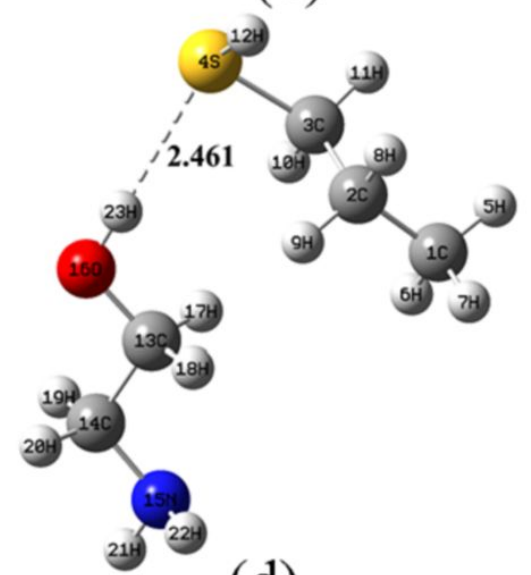

(d)

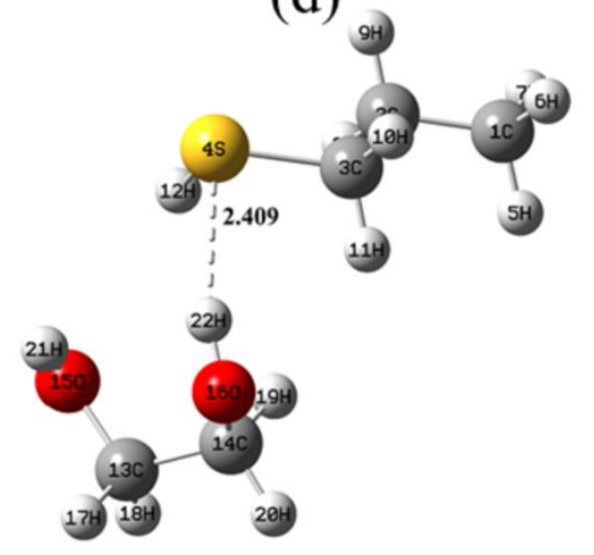

(f)

Fig.1S. The most stable structures with minimum energy for (a) NPA-PM; (b) IPA-PM; (c) ET-PM; (d) MEA-PM;(e) MeOH-PM; (f) EG-PM dimers. 

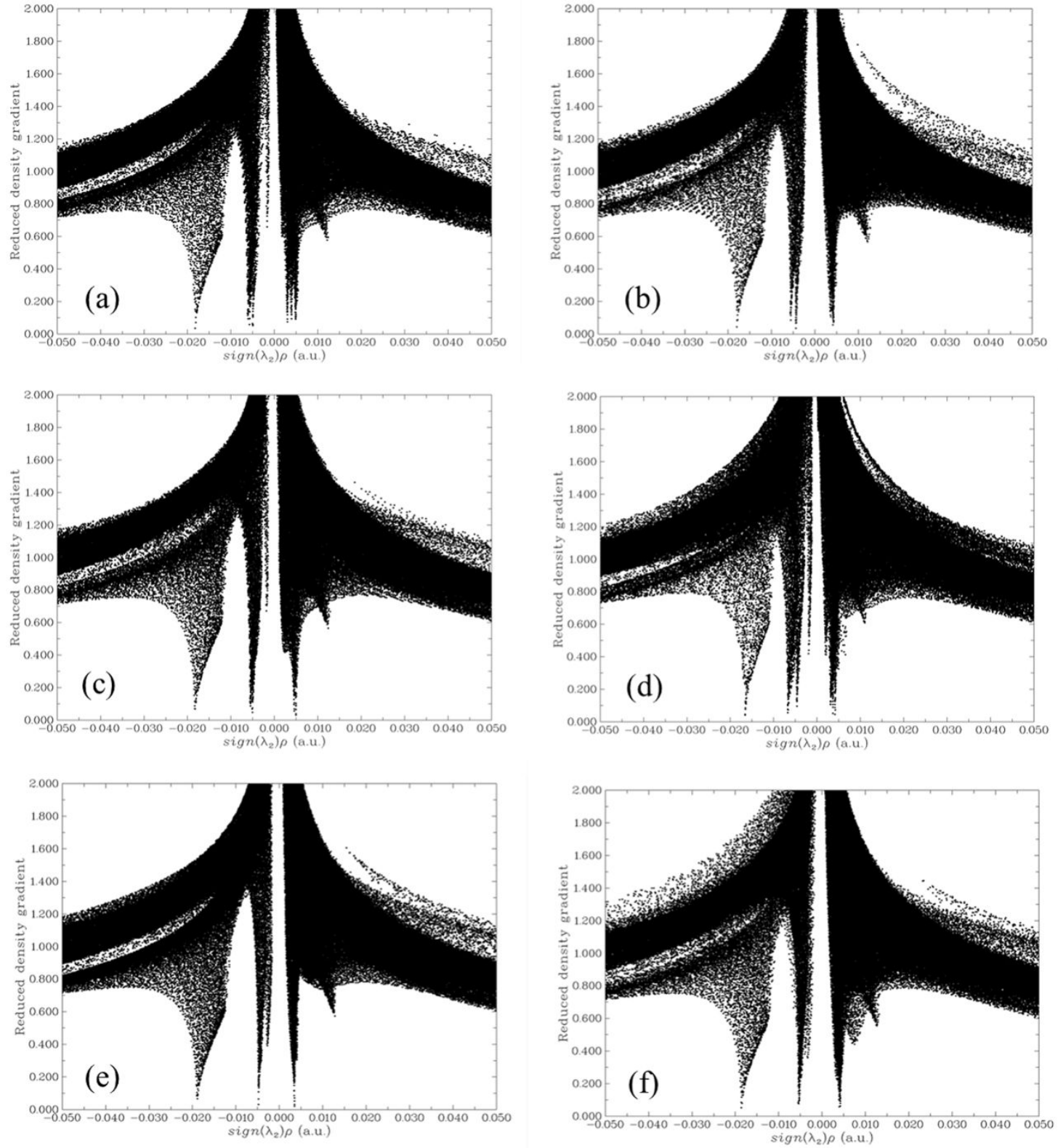

Fig.2S. Plot of $\operatorname{sign}\left(\lambda_{2}\right) \rho$ vs RDG gradient isosurfaces (right) for (a) NPA-PM; (b) IPA-PM; (c) ET-PM; (d) MEA-PM;(e) MeOH-PM; (f) EG-PM dimers. 


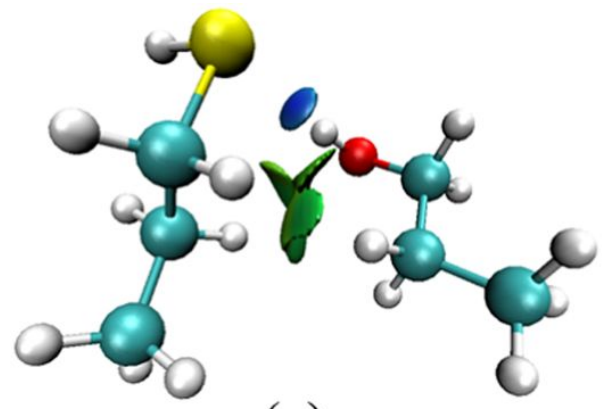

(a)

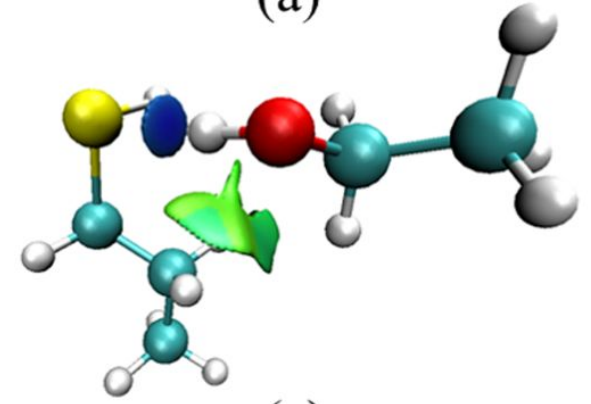

(c)

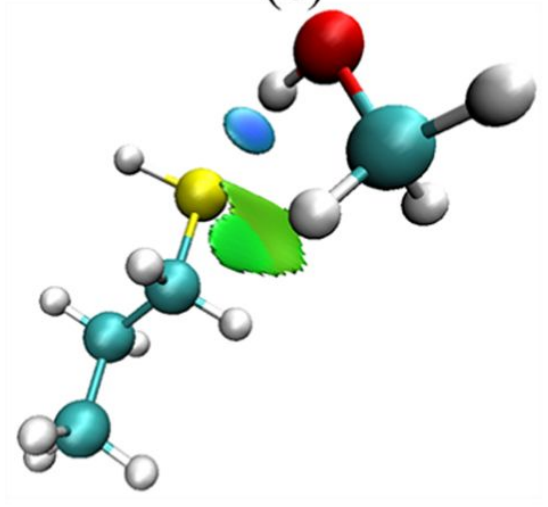

(e)

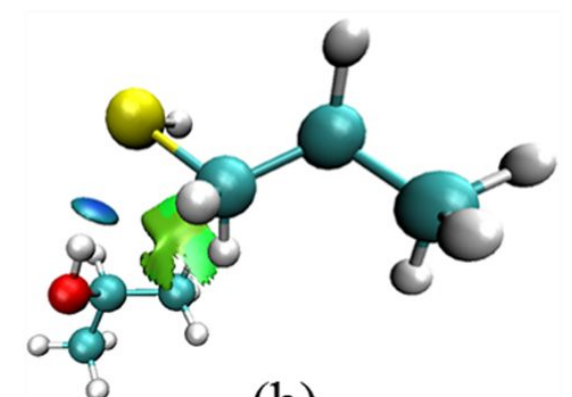

(b)

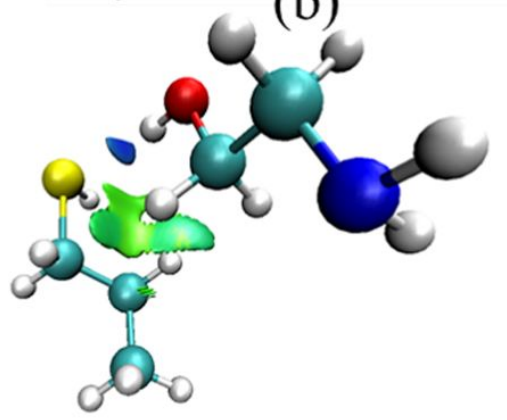

(d)

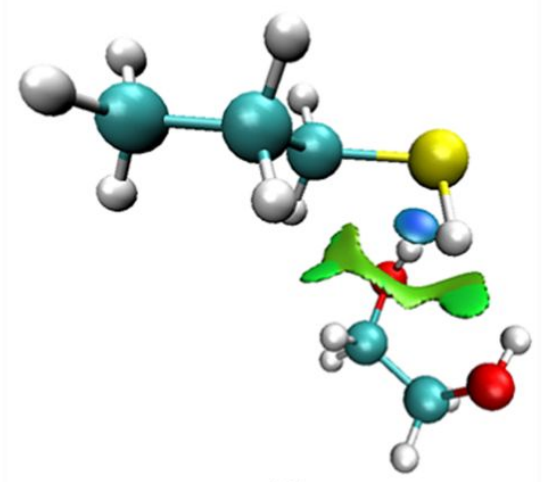

(f)

Fig.3S. Gradient isosurfaces for (a) NPA-PM; (b) IPA-PM; (c) ET-PM; (d) MEA-PM;(e) MeOH-PM; (f) EG-PM dimers. 


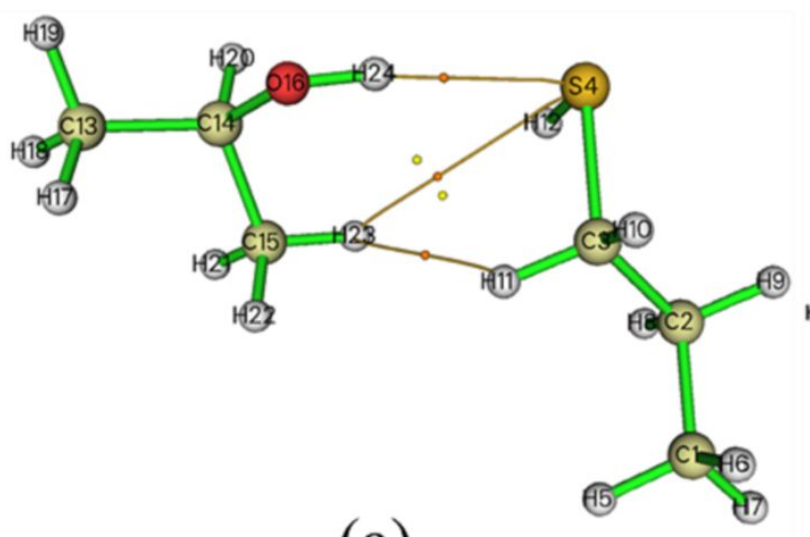

(a)

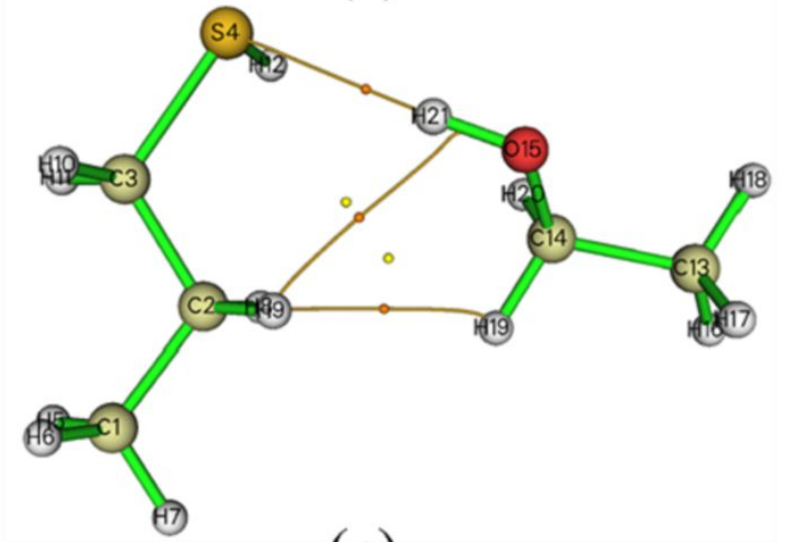

(c)

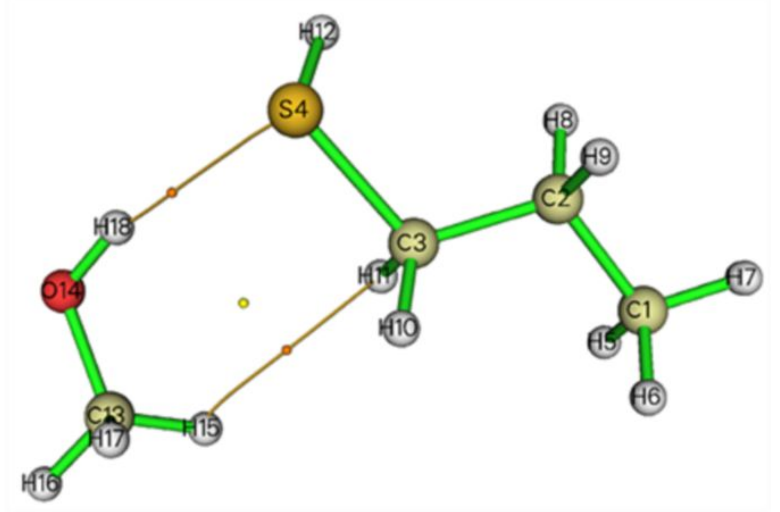

(e)

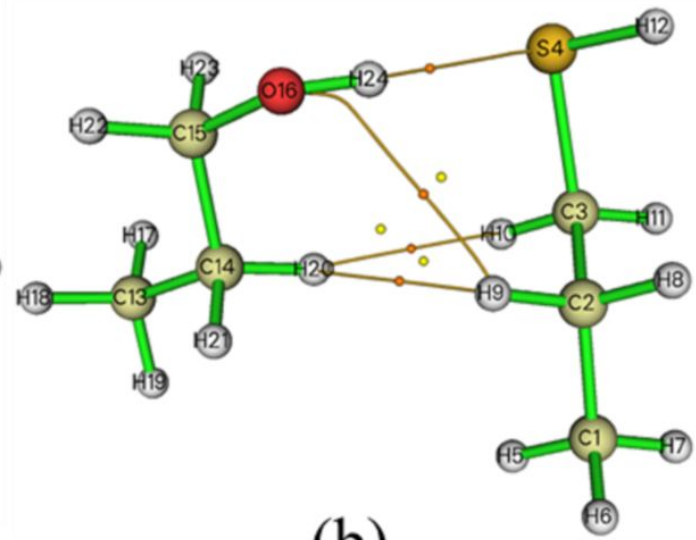

(b)

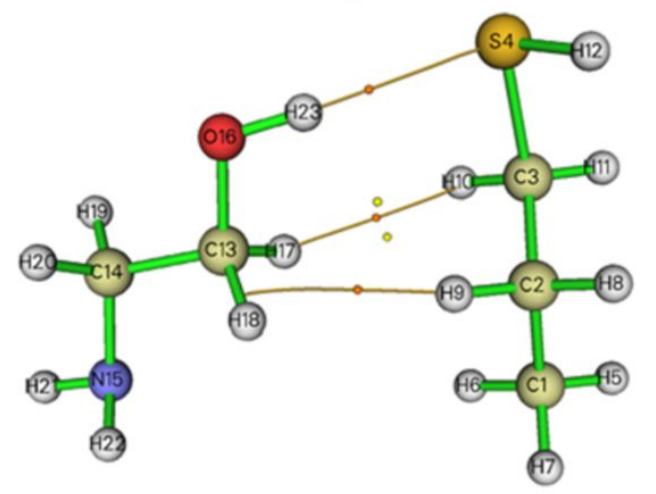

(d)

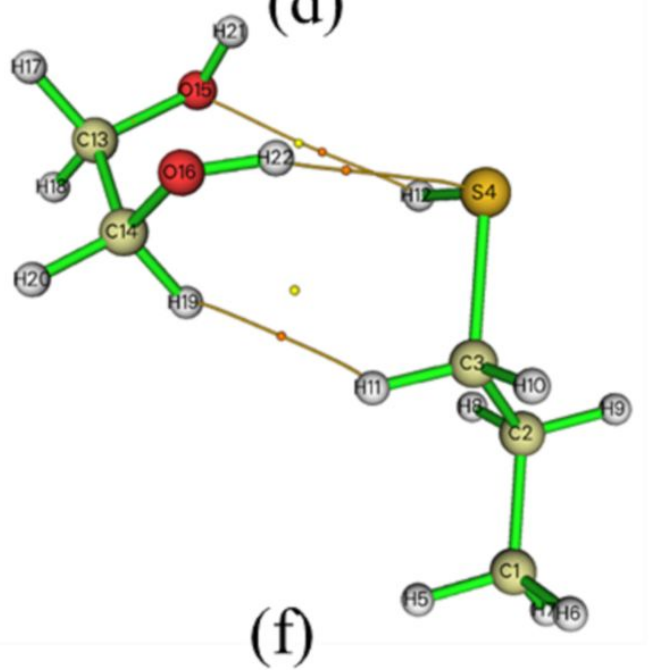

Fig. 4S. AIM molecular topography map showing BCPs distribution and bond paths for (a) NPA PM; (b) IPA - PM; (c) ET - PM; (d) MEA.-PM;(e) MeOH-PM; (f) EG-PM. Orange dots represent BCPs. 